\section{ECCOMAS}

\section{Proceedia}

COMPDYN 2021

$8^{\text {th }}$ ECCOMAS Thematic Conference on Computational Methods in Structural Dynamics and Earthquake Engineering

M. Papadrakakis, M. Fragiadakis (eds.) Streamed from Athens, Greece, 28 - 30 June 2021

\title{
CALIBRATION OF NUMERICAL MODELS TO SUPPORT SHM: THE CONSOLI PALACE OF GUBBIO, ITALY
}

\author{
Serena Cattari ${ }^{1}$, Daniele Sivori ${ }^{1 *}$, Sara Alfano ${ }^{1}$, Laura Ierimonti ${ }^{2}$, \\ Nicola Cavalagli ${ }^{2}$, Ilaria Venanzi ${ }^{2}$, Filippo Ubertini ${ }^{2}$ \\ ${ }^{1}$ University of Genoa, DICCA, Dept. of Civil, Chemical and Environmental Engineering \\ Via Montallegro 1, 16145, Genoa \\ serena.cattari@unige.it, daniele.sivori@dicca.unige.it, asara94@gmail.com \\ ${ }^{2}$ University of Perugia, DICA, Dept. of Civil and Environmental Engineering \\ Via G. Duranti 93, 06125, Perugia \\ laura.ierimonti@unipg.it, nicola.cavalagli@unipg.it, ilaria.venanzi@unipg.it, filippo.ubertini@unipg.it
}

\begin{abstract}
Historical masonry structures constitute a fundamental part of the built cultural heritage but are characterized by an intrinsic vulnerability to ageing and natural hazards, in particular to earthquakes. The related need to assess their current health condition and to ensure their future conservation is giving rise to increasing efforts in scientific research. The combined employment of health monitoring systems and structural modelling is widely adopted in this field, either to better interpret the effects of age-related degradation or to reliably predict the structural response to earthquakes. Both scenarios can leverage experimental measurements for the model calibration, thus reducing epistemic and aleatory uncertainties in the assessment phase. Among the available modelling strategies, refined Finite Element (FE) models represent the most common choice in the SHM perspective for monumental URM structures. Nonetheless, the computational effort required by the assessments in the nonlinear field - unavoidable in seismic evaluations - is often unfeasible, especially in practice engineering. In the case of palaces, an alternative is the employment of more computationally efficient formulations such as Equivalent Frame (EF) models. Within this framework, the paper firstly deals with the equivalent-frame modelling and model updating of the Consoli Palace, a historic masonry building in Gubbio (Italy) investigated through ambient vibration tests. The peculiar aspects of the building - e.g. the unusually high inter-storey height, the presence of vaulted floors, the irregular distribution of the openings - make the equivalent-frame idealization a challenging task. The comparison with a detailed finite element model developed in previous research points out the differences and limits of the two approaches, providing some suggestions to benefit from their integrated use.
\end{abstract}

Keywords: historical masonry buildings, Structural Health Monitoring, Equivalent Frame models. 


\section{INTRODUCTION}

Masonry structures constitute probably the largest part of the worldwide built heritage. Being masonry one of the oldest materials employed to build by humankind, this structural typology is commonly characterized by exceptional historical, cultural and architectural value. For these reasons, structural health assessment, conservation and rehabilitation of the built masonry heritage are crucial responsibilities of today's society. The statement is relevant both from a social and an economic point of view, urging for the efforts of the scientific community to address these tasks. Indeed, because of their age, old masonry structures suffer today the effects of ageing and deterioration over the past centuries, together with the succession of countless anthropic modifications and the constant acting of natural phenomena. These aspects, considering the intrinsic complexity of old masonry structures, make their structural health assessment a challenging goal.

In this context, the employment of non-destructive experimental techniques is leading the Structural Health Monitoring (SHM) research field, aimed at supporting the engineering judgment through experimental evidence. Together with the measurement of environmental factors, a primary role is played by dynamic testing through permanent monitoring systems, allowing for real-time identification and tracking of the dynamic properties of existing structures. Dynamic properties, indeed, are those commonly assumed as the proxy of structural health, so that detection of their variations corresponds to the potential - such as the occurrence of damage.

If the main trends of the research [1] follow the employment of data-driven statistical methodologies aimed at unsupervised evaluations, not less important is the complementary employment of engineering-based model-driven approaches, relying on accurate mechanical models for the simulation of the structural behaviour. In the case of historical masonry structures, among the possible modelling strategies (reviewed in [2][3] and discussed in [4] from a seismic engineering perspective), the common approach relies on refined Finite Element (FE) models, allowing for a detailed idealization of the structure. Such a potential, commonly, comes at the cost of (i) a large set of input parameters and (ii) high computational requirements, which often makes the employment of FE models unfeasible - for example, in nonlinear seismic analyses.

In the case of palaces, on the other hand, an alternative option is provided by Equivalent Frame (EF) models, specifically addressed to the analysis of the global seismic response of ordinary masonry buildings. This modelling technique discretizes the masonry continuum as an assemblage of macroscopic structural elements. Being governed by few mechanical parameters and a limited number of elements, EF models are an attractive choice thanks to their computational efficiency [5]. Nonetheless, as discussed in the following Sections, the common simplifying assumptions of this approach challenge its applicability in the case of monumental masonry palaces.

Based on the above presented motivations, the paper addresses the employment of EF models to support the SHM in the case of masonry palaces, proposing some preliminary considerations through the comparison with more refined FE models. In particular, the historical Consoli Palace of Gubbio, Italy, is chosen as the suited case study ( $\$ 2)$. The three-dimensional FE of the palace, previously developed and dynamically calibrated by the University of Perugia research group, is assumed as the reference result (\$3.2). An EF formulation of the palace is proposed, highlighting some possible issues related to common modelling assumptions ( $\$ 4.1)$. The model is dynamically calibrated based on the results of AVTs employing a global search algorithm, taking into account the uncertainties related to the limited knowledge of the mechanical properties of the existing structure, as well as those produced by the EF idealization $(\S 4.2)$. The dynamics of the updated model is satisfyingly close to the experimental evidence and the set of optimal parameter points out the importance of the out-of-plane stiffness contribution of 
masonry walls ( $\$ 4.3)$. A final comparison between the updated models shows good accordance in terms of dynamic and seismic properties (§4.4), confirming the possibility of their integrated use in the SHM perspective and pointing out interesting developments of the research $(\S 5)$.

\section{THE CONSOLI PALACE OF GUBBIO, ITALY}

The Consoli Palace (Figure 1a), located in the medieval town of Gubbio (Central Italy) is a monumental palace built between 1332 and 1349 according to different architectural building styles. The whole structure (Figure 1b) is mainly made of calcareous stone masonry also in the newest parts, as the staircase of the main façade, which is characterized, in the upper part, by coupled round-arched windows separated by lesenes. Above them, merlons enclose the rooftop and the bell tower. The lower part of the main façade is characterized by mullioned windows, a Gothic-style portal and the main staircase.

The palace has a 40x20 m rectangular plan and an elevation of about $60 \mathrm{~m}$. The structure is arranged on different floors: two levels on the foundation system; the Arengo hall at a height of $4.64 \mathrm{~m}$, accessible from the principal staircase (Figure 1c); the Nobili hall at a height of 18.89 $\mathrm{m}$ (Figure 1d); the rooftop at a height of about $30 \mathrm{~m}$. The Arengo hall, in particular, develops seamlessly - i.e. without intermediate walls - thanks to the huge structural thickness of the perimetral walls, which support a huge barrel vault. The Southside of the Palace is characterized by the loggia, a not-in-built architectural portion.

A recent in situ survey has allowed identifying different connection degrees between structural elements (walls-to-walls, walls-to-vaults) standing from interlocking to simply leaning elements. Moreover, the external stones of the Palace exhibit an apparent state of degradation, possibly attributed to exposition to environmental stresses and pollutants, such as biological patina, black crust, presence of vegetation, spalling, reintegration and encrustation.

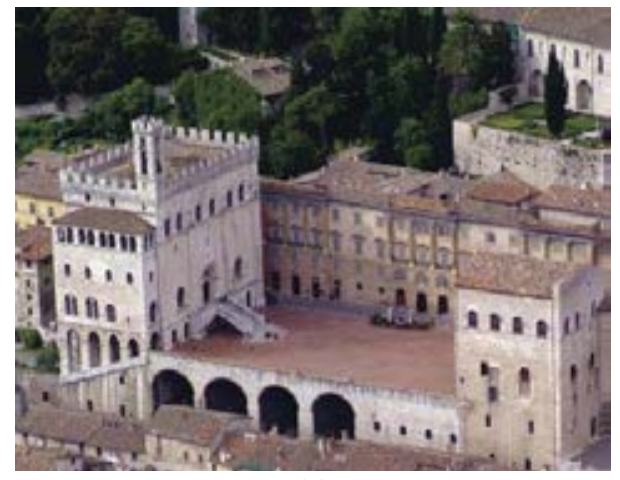

(a)

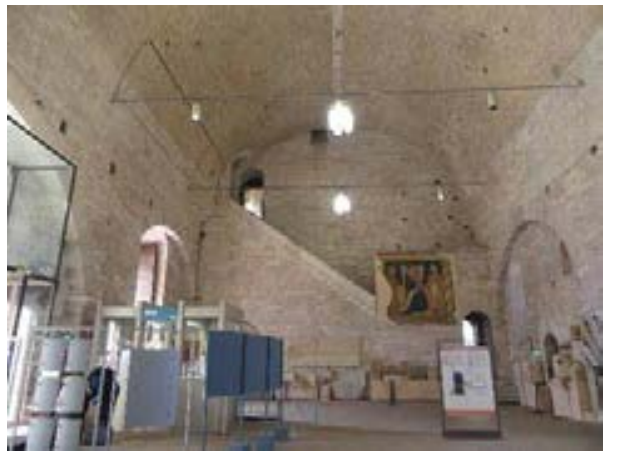

(c)

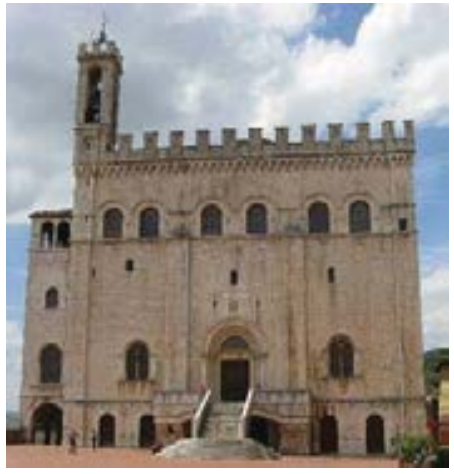

(b)

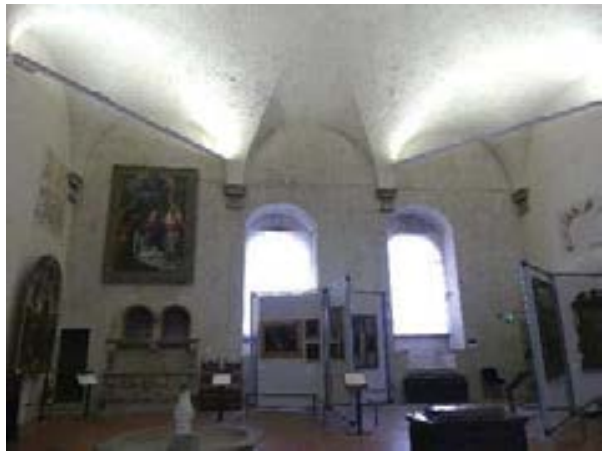

(d)

Figure 1: The Consoli Palace of Gubbio, Italy. (a) Aerial view of the Consoli Palace and the main square "Piazza Grande", (b) main façade, (c) Arengo hall and (d) Nobili hall. 


\section{TARGET DYNAMIC DATA AVAILABLE FOR MODELS' CALIBRATION AND COMPARISON}

\subsection{Dynamic identification from Ambient Vibration Tests (AVTs)}

The dynamic properties of the Consoli Palace were investigated through Ambient Vibration Tests (AVTs) carried out on May $4^{\text {th }}, 2017$. The main purpose of the AVTs was the identification of the elastic dynamic properties of the structure, i.e., natural frequencies, mode shapes and damping ratios, for structural monitoring and finite element model calibration purposes.

According to the hypothesis of in-plane rigid behaviour of the horizontal floor diaphragms, a network of 9 uni-axial high sensitivity piezoelectric accelerometers - model PCB 393B12, capable of measuring maximum accelerations of $\pm 0.50 \mathrm{~g}$ with a sensitivity of $10 \mathrm{~V} / \mathrm{g}$ - were deployed on the three main floors of the Palace (rooftop, Arengo hall, Nobili hall, Figure 2).

The AVT was conducted by using a multi-channel data acquisition system characterized by 24-bit resolution, $102 \mathrm{~dB}$ dynamic range and anti-aliasing filters.

The modal identification of Consoli Palace was performed by considering accelerations induced by micro-tremors, mainly due to wind and weak traffic excitation coming from the neighbouring area around the Palace. Consecutive time windows of $1800 \mathrm{~s}$ were collected, corresponding to more than 4000 times the fundamental period of the building.

The acceleration responses of the palace were recorded with the hardware own sampling frequency of $1652 \mathrm{~Hz}$. Then, since the dominant natural frequencies were expected to lay below $10 \mathrm{~Hz}$, signals were down-sampled to $40 \mathrm{~Hz}$ after low-pass filtering, with a Nyquist frequency of $20 \mathrm{~Hz}$.

The acquired signals were affected by anomalous peaks due to the bells-dependent nonstationary excitation, produced by the bell tower playing all day and night long with a 15-minute step interval. To remove this effect, an automated simple pre-processing tool has been implemented and applied to the data enabling to identify portions of the signals containing peak response accelerations overcoming a prescribed threshold (assumed as ten times the root mean square value of the signal) and to remove these portions - after application of half Hanning windows to the tails of the signals to be concatenated.

Finally, the dynamic identification was performed employing the Stochastic Subspace Identification (SSI) technique. To briefly introduce the SSI methods, it is convenient to consider a time-invariant $n$-dof structural system, whose state-space equations of motion can be written as

$$
\begin{gathered}
\boldsymbol{x}(k+1)=\boldsymbol{A} \boldsymbol{x}(k)+\boldsymbol{w}(k) \\
\boldsymbol{y}(k)=\boldsymbol{C} \boldsymbol{x}(k)+\boldsymbol{v}(k)
\end{gathered}
$$

where $k$ denotes the time step; $\boldsymbol{x} \in \mathbb{R}^{n}$ is the state vector; $\boldsymbol{y} \in \mathbb{R}^{l}$ is the vector of the $l$ output measurements; $\boldsymbol{w} \in \mathbb{R}^{n}$ is the external input, modelled as a white noise process vector; $\boldsymbol{v} \in \mathbb{R}^{l}$ is a white noise affecting the measurements; $\boldsymbol{A} \in \mathbb{R}^{n \times n}$ is the system matrix; $\boldsymbol{C} \in \mathbb{R}^{l \times n}$ is the output matrix. Then, thanks to a transformation of the state-space system, rewritten in the frequency domain by its transfer function through the so-called Canonical Variate Analysis (CVA) algorithm, the modal features can be extracted. More extensive details on this modal identification tool are reported in [6]. Table 1 summarizes the AVT-based fundamental vibration modes, where directions $x$ and $y$ are defined along the larger and the smaller dimension of the rectangular plan, respectively. The global mode shapes, i.e., 1-2-5, identified by the adopted SSI procedure are presented in Figure 3. 


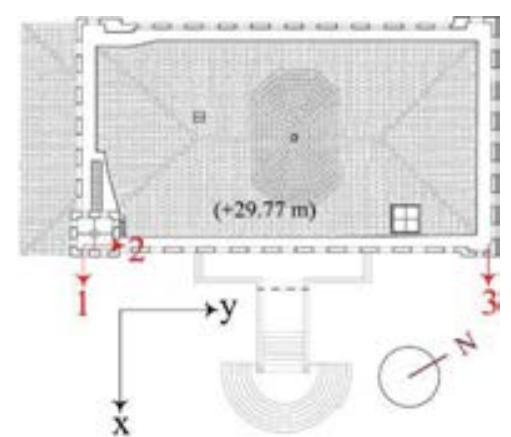

(a)

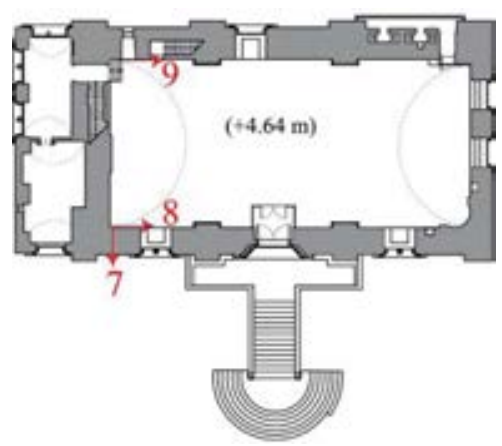

(b)

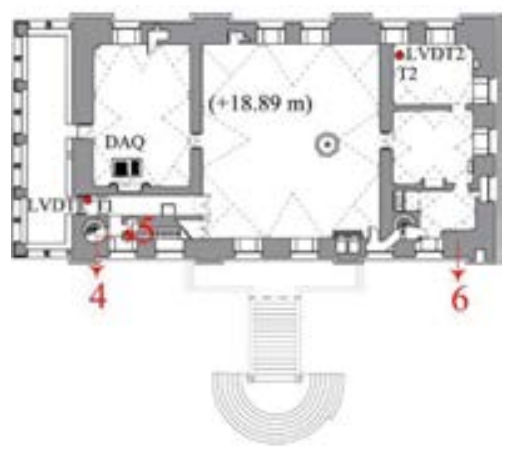

(c)

Figure 2: Configuration of 9 accelerometers and data acquisition system adopted in the AVT (a) at the top of the building (channels 1, 2, 3), (b) in the Arengo hall (channels 7, 8, 9), (c) in the Nobili hall (channels 4, 5, 6).

\begin{tabular}{lllll}
\hline Mode & Type & Description & $f_{e}(\mathrm{~Hz})$ & $\xi_{e}(\%)$ \\
\hline 1 & global & flexural along $x$-direction & 2.296 & 1.121 \\
2 & global & flexural along $y$-direction & 2.989 & 0.751 \\
3 & local & flexural of the bell tower & 3.508 & 0.779 \\
4 & local & flexural of the bell tower & 3.743 & 2.477 \\
5 & global & torsional & 4.172 & 1.104 \\
6 & local & torsional of the bell tower & 7.035 & 1.089 \\
\hline
\end{tabular}

Table 1: Identified natural frequencies $\left(f_{e}\right)$ and damping ratios $\left(\xi_{e}\right)$ of the Palace, estimated by SSI of the AVTs carried out on May 4th 2017.

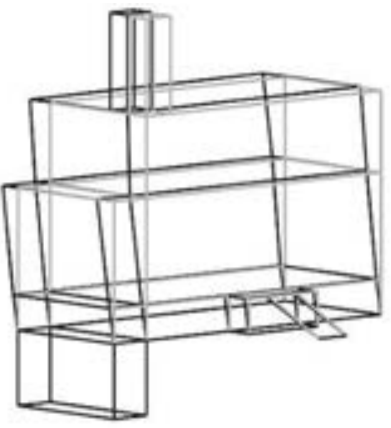

(a)

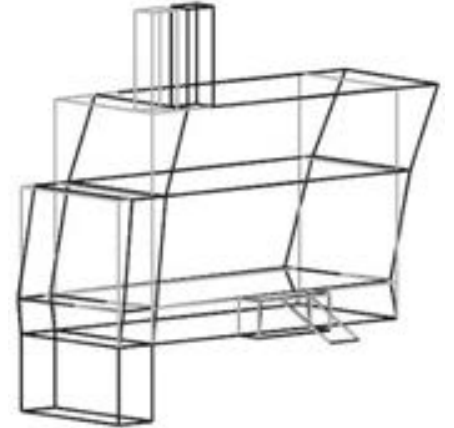

(b)

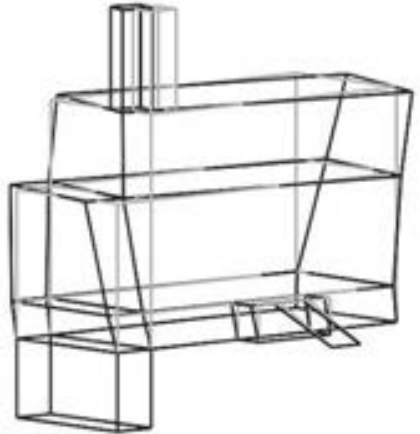

(c)

Figure 3: Global mode shapes of Consoli Palace identified from AVT acceleration records by automated SSI.

(a) Mode 1, (b) mode 2 and (c) mode 5.

\subsection{Updated Finite Element (FE) model of the palace}

To perform a consistent interpretation of the identified vibration modes, a 3D numerical Finite Element (FE) model of the structure has been built in the ABAQUS environment [7]. The mesh of the model is composed of a dense grid of tetrahedral elements (see Figure 5a in $\S 4.1$ ). Based on the principal architectural portions identified within the building (as described in §2), the model has been subdivided into four homogenous parts characterized by different values of Young's modulus of the masonry: the foundation level, the Arengo level, the Nobili level and the bell-tower. Hence, Young's moduli are assumed as uncertain parameters and their values have been adjusted in the calibration process to minimize the average relative difference 
between numerically predicted and experimentally identified natural frequencies. On the contrary, the same values of Poisson's coefficient and material density have been considered in the whole building.

The updated values of Young's moduli are summarized in Table 2. The relative difference $\Delta f$ between experimentally identified $\left(f_{e}\right)$ and numerically predicted $\left(f_{n, F E}\right)$ natural frequencies is highlighted in Table 3. Extensive details on the FE model calibration can be found in [8].

\begin{tabular}{lll}
\hline Part & Initial value $(\mathrm{MPa})$ & Updated value $(\mathrm{MPa})$ \\
\hline Foundation level & 2440 & 3327 \\
Arengo level & 2440 & 3510 \\
Nobili level & 2440 & 3327 \\
Bell tower & 2440 & 3450 \\
\hline
\end{tabular}

Table 2: Updated values of Young's moduli.

\begin{tabular}{llll}
\hline Mode & $f_{e}(\mathrm{~Hz})$ & $f_{n, F E}(\mathrm{~Hz})$ & $\Delta_{f}$ \\
\hline 1 & 2.296 & 2.352 & 0.024 \\
2 & 2.989 & 3.047 & 0.019 \\
3 & 3.508 & 3.460 & -0.014 \\
4 & 3.743 & 3.544 & -0.053 \\
5 & 4.172 & 4.171 & $<0.001$ \\
6 & 7.035 & 7.09 & 0.008 \\
\hline
\end{tabular}

Table 3: Comparison between experimentally identified $\left(f_{e}\right)$ and numerically predicted $\left(f_{n, F E}\right)$ natural frequencies, where $\Delta_{f}=\left(f_{n}-f_{e}\right) / f_{e}$ represents the relative frequency difference.

\section{FORMULATION AND CALIBRATION OF THE EQUIVALENT FRAME (EF) MODEL}

\subsection{Equivalent-frame modelling assumptions and related issues}

The structural model of the Consoli Palace, based on the Equivalent Frame (EF) modelling approach, is built with the commercial software 3Muri (Release 11.5.7) distributed by S.T.A. Data. In the EF approach, as known, the deformability and the nonlinearity are concentrated in specific parts of the masonry walls, i.e. piers and spandrels. These are the portions in which seismic damage tends to concentrate, as widely testified by in situ observations of existing buildings hit by recent earthquakes (for example, in Italy [9][10][11][12]). According to this idealization, the piers - vertical portions of masonry between horizontally aligned openings resist both vertical and horizontal loads, whereas the spandrels - horizontal portions of masonry between vertically aligned openings - play a primary role in piers coupling. The remaining portions of the walls are idealized as rigid nodes.

The EF approach, thus, is commonly employed to simulate the global seismic response of ordinary masonry buildings. Some of the simplifying assumptions adopted by EF models, on the other hand, make their application more involved - and sometimes unsuitable - for historical monumental buildings. This is the case of the Consoli Palace, for which the EF approach requires particular caution and poses interesting engineering challenges. The following paragraphs address some of the issues faced in the synthetic EF description of the palace, highlighting the differences with more detailed FE approaches and proposing some possible modelling solutions. 


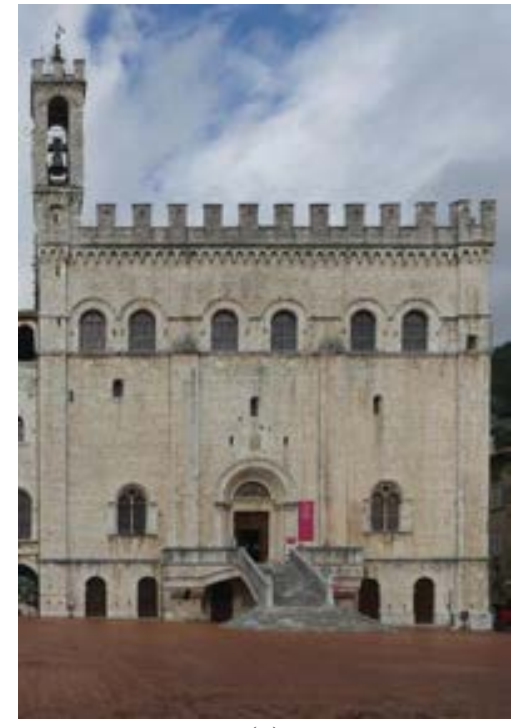

(a)

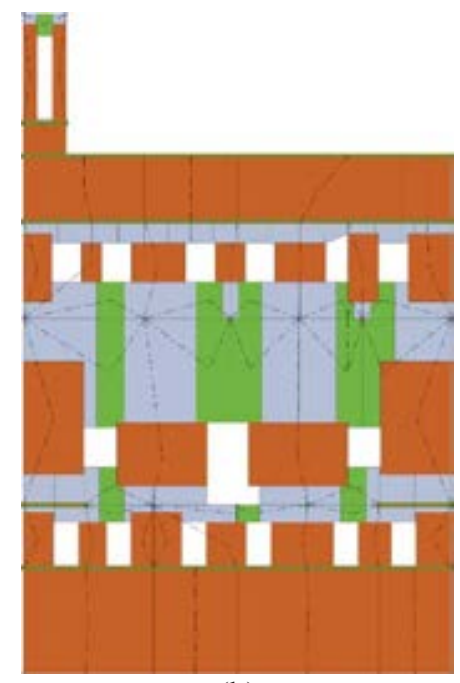

(b)

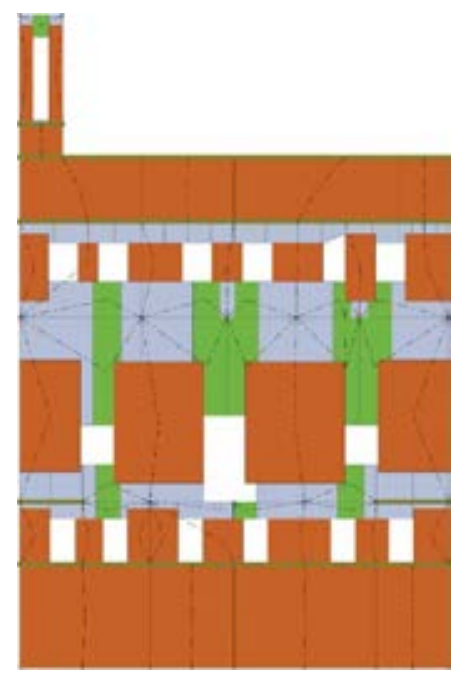

(c)

Figure 4: (a) Rectified picture of the Consoli Palace façade. Corresponding EF mesh according to (b) the criteria implemented in 3Muri and (c) after manual modification. The colours orange, green and light blue represents respectively piers, spandrels and rigid nodes.

The definition of the geometry of the vertical structural elements (Figure 4), mainly depends on the number and arrangement of openings. This procedure is straightforward when openings are aligned and regularly distributed. Conversely, in the case of irregularly shaped and sparse openings, the EF modelling becomes non-trivial and requires expert engineering judgment due to the potential source of large dispersion in the simulation of the seismic response [13][14]. Indeed, the structural mesh significantly influences the static and dynamic behaviour of the structure in the linear and nonlinear regime, so that appropriate criteria for its definition are crucial to obtain reliable simulations.

Several contributions of the literature address this task, providing various indications [15][16] [17][18] for the definition of the EF mesh geometry. Nonetheless, most of the rules have been developed based on a regular arrangement of the openings. Among those, recent research [19] and detailed comparisons with cracking patterns of damaged buildings [14] suggest the criterion implemented by 3Muri software to be reliable. Defining the effective height of piers is particularly challenging when the layout of the openings is irregular, which unfortunately represents a recurrent situation in existing buildings. In recent years, many researchers have focused their efforts to overcome this issue (see for example [20][21][22][23][24][25]). However, no shared consensus in the scientific community and standardized rules have been established yet.

The openings of the Consoli Palace are often irregularly arranged on the walls, without systematic horizontal and vertical alignments. In this case, according to the criterion adopted by 3Muri software, large portions of the walls would be idealized as rigid nodes due to the unusually large interstorey heights (such as those of the Arengo hall, §2). In light of these issues, firstly, some very small openings on the perimeter of the palace have been neglected to enhance the quality of the meshing. As shown by recent studies [25][26], this simplification is acceptable when the opening is sufficiently smaller than the masonry panel. Secondly, the software-generated mesh (Figure 4b) has been carefully revised and manually edited, to define deformable masonry portions more consistent with physical expectations (Figure 4c).

For what concerns the floor diaphragms, they are modelled as rectangular orthotropic membranes [15]. Their elastic mechanical properties are identified by the axial Young's moduli of 
the two main directions of in-plane deformation and, more importantly, by the shear modulus $G_{d}$. This parameter, depending on the diaphragm thickness, governs the equivalent shear stiffness provided by floor diaphragms to the coupled walls, determining the redistribution of horizontal seismic actions as well. As previously highlighted (\$2), the Consoli Palace is mainly characterized by vaulted floors of different typologies. Their geometric characteristics are peculiar, especially those regarding the huge barrel vault covering the Arengo hall, in which narrow and long vaulted rooms have been built within the volume of the vault itself. This complex system of rooms has not been explicitly described in the EF model, coherently with its simplified nature. Nevertheless, the dynamic calibration of the model considers wider uncertainties in the masses and horizontal stiffness contributions provided by this floor (see $\S 4.2$ ).

With similar reasoning and due to its complexity, the roofing system has been modelled only through its mass contribution. It should be remarked that the proper and additional masses of floors, in the EF model, are distributed to the diaphragm perimeter nodes. In the case of the Consoli Palace, these masses are relevant, accounting for $30 \%$ of the total mass of the structure.

Another important aspect of the three-dimensional EF assembling regards the degree of connection between walls and horizontal diaphragms and between walls. The first is governed by the stiffness properties of the diaphragms themselves. The second, on the other hand, must reproduce the quality of the anchoring between intersecting walls, depending on the mechanical properties of masonry and the stone arrangement at the walls joint. Different effectiveness of the wall-to-wall connection may be achieved by different modelling strategies in EF models, as discussed in [27], among which the use of connecting beams is particularly effective.

The recent in situ survey $(\S 2)$ allowed identifying those walls in which the so-called flange effect is guaranteed, so to be equivalently described by stiff coupling beams. In other cases, walls are not intersecting but simply leaning against each other, so that the flange effect is negligible. The intermediate cases are reproduced in the model through appropriate connecting beams, whose stiffness properties determine the degree of coupling between intersecting masonry walls.

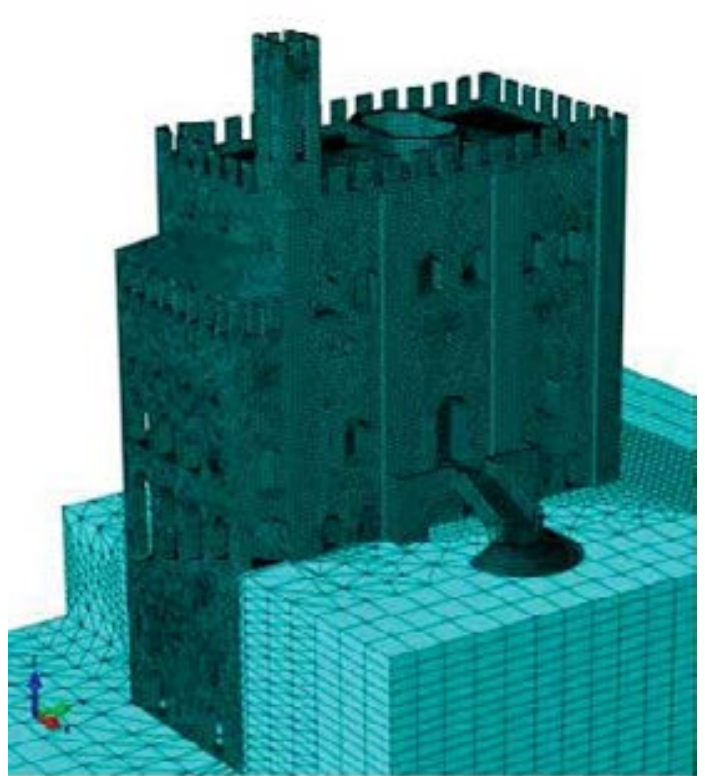

(a)

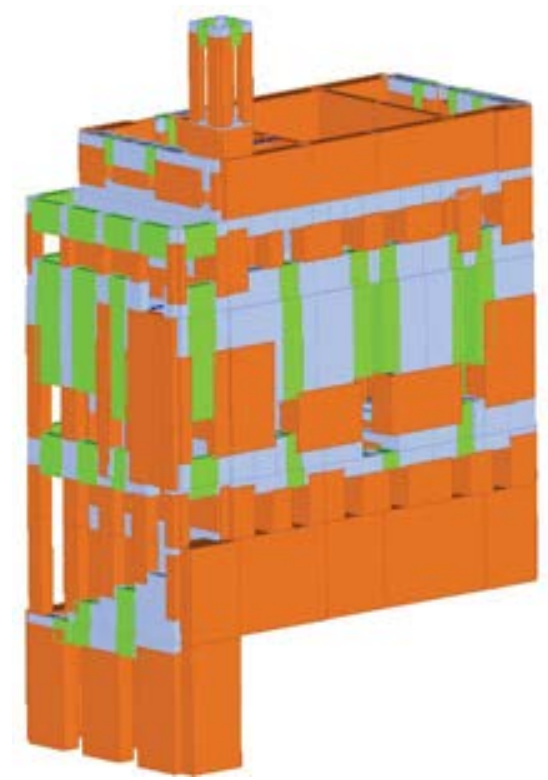

(b)

Figure 5: (a) Finite element (FE) model and (b) Equivalent Frame (EF) model of the Consoli Palace. 
Finally, the three-dimensional structure is assembled from bi-dimensional elements, walls elements and floor diaphragms, neglecting their out-of-plane stiffness contributions. This assumption is reasonable and widely adopted in the analysis of ordinary masonry buildings. In the case of Consoli Palace, however, the thicknesses of bearing walls are much larger - sometimes comparable with the in-plane extension, see $\$ 2$ and Figure 3 - than those found in ordinary buildings. As a consequence, their out-of-plane stiffness is expected to influence significantly the global dynamic behaviour of the structure. This effect has been properly accounted for in the calibration process (see $\S 4.2$ ).

In light of these premises, the resulting three-dimensional EF model (Figure 5b) faithfully reproduces the geometric and structural characteristics of the palace, derived from the available documentation and refined following the on-site investigations. If compared to the FE model (Figure 5a), indeed, the biggest simplification lies in the impossibility to explicitly model the volume of the subsoil at the foundation level. In the EF model, this limitation is overcome by employing proper node constraints.

\subsection{Uncertainties and updating technique}

The model updating process is formulated as a constrained global optimization problem, in which uncertain mechanical parameters characterizing the structural model are calibrated to minimize the differences between the numerical simulations and the experimental results. Considering the availability of three reliable experimental modes - identified from AVTs in $\$ 3.1$ and employed for the calibration of the FE model of the palace in $\S 3.2$ - resulting in three target frequencies and as many mode shapes, the dimensions of the problem (i.e. the number of free parameters) are limited to a maximum of six unknowns.

The extension of the parameter space, i.e. allowed range of variation of each parameter, reflects (i) the related degree of uncertainty deriving from the limited knowledge of the existing structure and (ii) as thoroughly discussed in the following, some limitations of the EF in modelling such a complex masonry structure (§4.1). Based on this premise, in a preliminary phase, five mechanical parameters - expected to influence the undamped free vibrations of the structure in the investigated range of frequencies - are assumed as the problem unknowns. Their reference values and ranges of variation are synthetically reported in Table 4.

The parameter $E_{m}$ represents the masonry Young's modulus of the whole body of the palace. According to the EF idealization, this quantity governs the in-plane flexural response of the vertical structural elements (i.e. piers and spandrels). In this respect, this parameter is expected to have a primary influence on the natural frequencies of the global (in this case, translational and rotational) modes. Since no experimental tests are available to directly characterize the mechanical properties of the palace masonry, the reference elastic modulus is assumed as the average value of the range proposed by the Italian Circular No. 7/2019 [28] for massive stone (Table C8.5.I) - which characterizes most of the palace bearing walls - taking into account the good quality of the mortar joint observed during the in situ surveys (Table C8.5.II). Similarly, concerning the lowest acceptable value, the range of variation is constrained according to the same code provision. Conversely, the less stringent upper bound is mainly aimed at an indirect in-plane compensation of the unmodeled out-of-plane stiffness of the vertical elements $(\S 4.1)$. This statement should not be surprising, observing the significant out-of-plane thickness of the bearing walls (\$2). The in-plane shear contribution, governed by the shear modulus of the masonry $G_{m}$, is derived assuming a Poisson ratio equal to 0.2 - as in the FE calibration (§3.2) leading to $G_{m}=E_{m} / 1.4$. 


\begin{tabular}{|c|c|c|c|c|}
\hline Parameter & Description & $\begin{array}{l}\text { Reference } \\
\text { value }\end{array}$ & $\begin{array}{l}\text { Lower } \\
\text { bound }\end{array}$ & $\begin{array}{l}\text { Upper } \\
\text { bound }\end{array}$ \\
\hline$E_{m}(\mathrm{MPa})$ & Young's modulus of palace masonry & 3420 & 2400 & 12000 \\
\hline$E_{m t}(\mathrm{MPa})$ & $\begin{array}{l}\text { Young's modulus of bell tower ma- } \\
\text { sonry }\end{array}$ & 3420 & 2400 & 12000 \\
\hline$\rho_{m}\left(\mathrm{~kg} / \mathrm{m}^{3}\right)$ & Mass density of palace masonry & 2200 & 1800 & 2400 \\
\hline$c_{M d}$ & $\begin{array}{l}\text { Relative variation of floor masses } \\
\text { (Arengo and roof level) }\end{array}$ & 1 & 0.5 & 1.5 \\
\hline$c_{G d}$ & $\begin{array}{l}\text { Relative variation of floor diaphragms } \\
\text { in-plane shear stiffness (vaults) }\end{array}$ & 1 & 0.5 & 25 \\
\hline$c_{\text {oop }}$ & $\begin{array}{l}\text { Relative variation of } E_{m} \\
\text { along the } y \text { direction }\end{array}$ & 1 & 0.5 & 1.5 \\
\hline
\end{tabular}

Table 4: Reference values and variation boundaries of the parameters calibrated in the updating process.

The Young's modulus of bell tower masonry $E_{m t}$ governs the frequency of the local modes of the bell tower, in particular those involving its flexural behaviour. From the mechanical point of view, no significant differences are expected between the palace and tower masonries - sharing the same typology, see $\S 4.1$. Choosing this parameter as a separate variable, nevertheless, allows decoupling the effects of the unmodeled out-of-plane stiffness belonging to the main building from those belonging to the tower itself. This parameter is expected to be overestimated from the updating procedure as well, due to the significant thickness of the pillars supporting the bell tower.

The variation of the mass density $\rho_{m}$ of the palace masonry is constrained according to a limited uncertainty related to the building material properties. It should be remarked, in this regard, that the wall mass contribution is around $70 \%$ of the total mass of the building, making the calibration process quite sensitive even to small variations of this parameter.

The relative weighting factor $c_{M d}$ takes into account the uncertainty in the definition of the proper and added masses insisting on the Arengo vault and the roof level. This parameter addresses the vertical loads contribution (i) the complex system of rooms rising above the Arengo vault and of (ii) the roofing, none of which being explicitly described by the model (§4.1). Such masses are estimated to be around 2000 ton each, making up around $60 \%$ of the floor masses and $15 \%$ of the total mass of the palace. According to the EF modelling assumptions, the diaphragms masses are lumped to the nodes located on the supporting perimeter walls, so that their variation is expected to influence the torsional inertia of the floors.

The coefficient $c_{G d}$ allows tuning the equivalent in-plane shear stiffness $G_{d}$ provided by horizontal diaphragms, in particular by those representing vaulted floors. This choice is justified by the unusually large dimensions of the Arengo vault ( $\$ 2)$, which make analytical estimations - such as those presented in [29] and employed to estimate reference values - less reliable. A recent contribution of the literature allows to quantitatively identify the actual shear stiffness of floor diaphragms from ambient vibration data [30]. Nevertheless, in this specific case, the limited number of sensors and their suboptimal location prevent a successful identification [31]. For these reasons, a finer calibration of this parameter is delegated to the updating process.

Finally, the factor $c_{\text {oop }}$ characterizes the masonry Young's modulus of the bearing walls aligned with the $y$ axis, according to the expression $E_{m, y}=c_{o o p} E_{m}$. This parameter addresses the potential unbalance between the global out-of-plane stiffness provided by each direction. Based on simple geometric considerations, observing the huge thickness and length of the two perimeter walls developing along $x$, the out-of-plane contribution is expected to be prominent along $y$ (i.e. $c_{\text {oop }}>1$ ). 
The simplifying assumptions of the 3Muri EF implementation - discussed in detail in $\S 4.1$ - and, more in general, those of the equivalent frame formulation, surely make the modelling of a complex structure such as the Consoli Palace a challenging task. On the other hand, if compared to FE modelling, the synthetic EF formulation ensures much lower computational requirements. Taking advantage of this feature, the inverse updating problem is approached from a global search perspective, aimed at the extensive exploration of the parameter space.

In particular, a genetic algorithm is chosen among the several global optimization approaches suitable to carry out the updating process. This metaheuristic search, belonging to the larger class of evolutionary algorithms, mimics the natural selection process relying on biologically inspired operators such as selection, crossover and mutation. The initial population size is set to 200 members, sampled uniformly from the bounded parameter space. Parents are selected among the members of each population with the lowest objective values. To ensure diversity, each subsequent generation is composed of (i) ten elite members, those with the highest ranking from the previous generation, (ii) $80 \%$ of the remaining members generated from parents crossover - mixing different genes, i.e. parameters - and (iii) 20\% generated from parents mutations - applying random Gaussian noise to their parameters.

The single objective function $\Pi$ to be minimized is representative of the relative differences between simulated and experimental modes, according to the expression

$$
\begin{gathered}
\Pi=w_{f} \Pi_{f}+w_{s} \Pi_{s}, \quad w_{f}+w_{s}=1 \\
\Pi_{f}=\sum_{k}\left(\frac{f_{n, k}-f_{e, k}}{f_{e, k}}\right)^{2}, \quad \Pi_{s}=\sum_{k} \frac{\left(1-\sqrt{\mathrm{MAC}_{n e, k k}}\right)^{2}}{\mathrm{MAC}_{n e, k k}}
\end{gathered}
$$

where $\Pi_{f}, \Pi_{s}$ measure respectively the differences in natural frequencies and mode shapes [32] - whose relative importance is governed by the weighting factors $w_{f}, w_{s}$ - over the $k$ selected modes. The expressions of $\Pi_{f}$ and $\Pi_{s}$ are balanced in their relative magnitudes, so that the updating process is penalizing frequency differences when MAC [33] values are greater than 0.7. Based on this consideration, intrinsically addressing the higher robustness of experimentally identified frequencies compared to mode shapes, the weights $w_{f}, w_{s}$ are initially assumed to be both equal to 0.5 .

\subsection{Dynamic calibration based on experimental results}

Few are the contributions of the literature addressing the use of AVTs results to dynamically calibrate EF models, aimed at reducing the aforementioned epistemic and aleatory uncertainties intrinsic to the modelling process [34].

Considering the set of experimental modes identified by AVTs (\$3.1) and following the approach followed by the FE model calibration (\$3.2), the first, second and fifth identified modes (Table 1, Figure 3) are selected as the target of the updating process. Convergence - as an average relative variation in the best objective function lower than $10 \%$ - is obtained at the eleventh generation, corresponding to 2300 function evaluations (modal analyses solved with the TreMuri research version [15]). The solution is achieved in around 40 minutes, exploiting the multiprocessing capabilities of a modern quad-core CPU. This time is comparable with the one employed by the corresponding FE model to solve a single modal analysis.

A first calibration is pursued employing a synthetic EF model in which the bell tower is not explicitly modelled, i.e. addressing its inertial contribution only. The corresponding label w/oT stands for "without tower". The set of reference parameter, named w/oT0 (Table 5), produces (i) large underestimates of natural frequencies and (ii) for the second mode, a poor mode shape correlation (Table 6). In particular, the relative frequency differences $\Delta_{f}=\left(f_{n}-f_{e}\right) / f_{e}$ range 
from $-30 \%$ to $-50 \%$. This outcome was expected, given the importance of the unmodeled outof-plane stiffness of the bearing walls (§4.1). The calibration results (referred to as w/oT1) show that, despite significant improvements in the objective function, frequency differences remain large. Indeed, a better agreement could be achieved - for example, setting $w_{f}, w_{s}$ equal to 0.8 and 0.2 respectively - at the cost of completely losing the mode shape correlation of the second mode. Conversely, no set of parameters manages to improve such a correlation, due to the torsional component not observed in experimental results (Figure 6a).

If the explicit introduction of the bell tower - label $T$ - does not improve significantly the reference configuration $\mathrm{T} 0$, better results are obtained from the calibration of the tower stiffness properties. The calibration T1 achieves (i) frequency differences lower than 5\% for the first two modes and lower than $10 \%$ for the fifth mode and (ii) good mode shapes accordance, resulting in a reduction of the objective function of one order of magnitude (Table 7). Remarkably, the second mode shows a pure translational behaviour along the $x$-direction (Figure 6b), significantly improving the agreement with experimental observations. This outcome seems to confirm the primary role played by the bell tower and its non-negligible influence on the global dynamics of the palace.

The corresponding set of updated parameters, compared with their reference values, shows a large increase in Young's moduli of both the palace and bell tower masonries. In the case of tower's pillars, a simple manual estimation of the unmodeled out-of-plane stiffness - ranging from 1.25 to 1.5 times the corresponding in-plane stiffness for the cantilever and doubleclamped schemes - yields physically reasonable estimates of the elastic moduli. The parameters governing the wall and diaphragms masses show a reasonable decrease, compatible with the initial underestimation of natural frequencies. The coefficient governing the in-plane shear stiffness of floor diaphragms, close to its upper bound, suggest the underestimation of the stiffness properties of vaults $(\S 4.2)$.

\begin{tabular}{l|ll|lll}
\hline Parameter & w/oT0 & w/oT1 & T0 & T1 & T2 \\
\hline$E_{m}(\mathrm{MPa})$ & 3420 & 6450 & 3420 & 11960 & 7650 \\
$E_{m t}(\mathrm{MPa})$ & $/ /$ & $/ /$ & 3420 & 7000 & 9100 \\
$\rho_{m}\left(\mathrm{~kg} / \mathrm{m}^{3}\right)$ & 2200 & 1810 & 2200 & 1970 & 1893 \\
$c_{M d}$ & 1 & 1.29 & 1 & 0.5 & 0.5 \\
$c_{G d}$ & 1 & 24.8 & 1 & 24.9 & 24.4 \\
$c_{\text {oop }}$ & $/ /$ & $/ /$ & $/ /$ & $/ /$ & 1.5 \\
\hline
\end{tabular}

Table 5: Reference and updated values of the parameters calibrated by the updating process.

\begin{tabular}{|c|c|c|c|c|c|c|c|c|}
\hline & & \multicolumn{4}{|c|}{$\mathrm{w} / \mathrm{oT0}-\Pi=0.4628$} & \multicolumn{3}{|c|}{$\mathrm{w} / \mathrm{oT} 1-\Pi=0.1723$} \\
\hline Mode & $f_{e}(\mathrm{~Hz})$ & Mode & $f_{n, E F}(\mathrm{~Hz})$ & $\Delta_{f}$ & MAC & $f_{n, E F}(\mathrm{~Hz})$ & $\Delta_{f}$ & $\mathrm{MAC}$ \\
\hline 1 & 2.296 & 1 & 1.19 & -0.482 & 0.97 & 1.63 & -0.290 & 0.98 \\
\hline 2 & 2.989 & 2 & 1.93 & -0.354 & 0.42 & 2.63 & -0.120 & 0.53 \\
\hline 5 & 4.172 & 3 & 2.02 & -0.516 & 0.85 & 2.83 & -0.322 & 0.87 \\
\hline
\end{tabular}

Table 6: Comparison between the experimental results, the reference and the updated EF model w/oT.

\begin{tabular}{|c|c|c|c|c|c|c|c|c|c|c|c|}
\hline & & \multicolumn{4}{|c|}{ Т0 - П = 0.3999} & \multicolumn{3}{|c|}{$\mathrm{T} 1-\Pi=0.0200$} & \multicolumn{3}{|c|}{$\mathrm{T} 2-\Pi=0.0164$} \\
\hline Mode & $f_{e}(\mathrm{~Hz})$ & Mode & $f_{n, E F}(\mathrm{~Hz})$ & $\Delta_{f}$ & MAC & $f_{n, E F}(\mathrm{~Hz})$ & $\Delta_{f}$ & MAC & $f_{n, E F}(\mathrm{~Hz})$ & $\Delta_{f}$ & MAC \\
\hline 1 & 2.296 & 1 & 1.19 & -0.482 & 0.97 & 2.34 & 0.019 & 0.99 & 2.25 & -0.020 & 0.99 \\
\hline 2 & 2.989 & 2 & 1.89 & -0.368 & 0.47 & 3.06 & 0.024 & 0.80 & 3.19 & 0.067 & 0.80 \\
\hline 5 & 4.172 & 5 & 2.27 & -0.456 & 0.78 & 3.83 & -0.082 & 0.77 & 3.87 & -0.072 & 0.84 \\
\hline
\end{tabular}

Table 7: Comparison between the experimental results and the updated EF model T. 


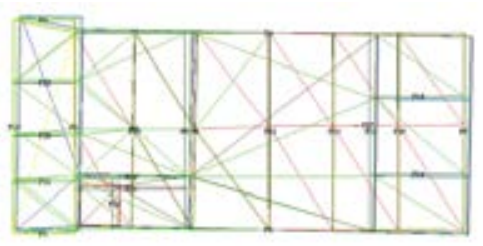

(a)

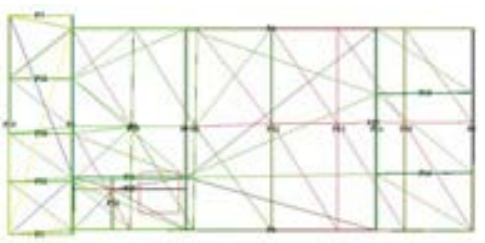

(b)

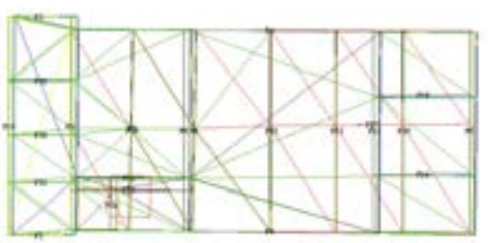

(c)

Figure 6: Shape of mode 2 in the (a) w/oT1, (b) T1 and (c) T2 updated EF models.

Introducing the coefficient $c_{\text {oop }}$, accounting for the directional effects of the unmodeled outof-plane stiffness (see $\S 4.2$ ), does not bring improvements to the updating process. Nevertheless, solution T2 gives some further insights into the model dynamic behaviour. Comparing T2 with $\mathrm{T} 1$, neglecting the small variations in mass density, a lower stiffness along the $x$-direction allows obtaining equally satisfying results. Conversely, a much higher stiffness along the $y$-direction - 1.5 times greater than that of $x$ - forms the optimal solution, confirming the expectations regarding the unbalance between the out-of-plane contributions provided by each direction.

\subsection{Comparison with the updated FE model}

The results of the model update process $(\S 4.2)$ show the ability of the calibrated EF model to correctly reproduce the experimental dynamics of the Consoli Palace. The unusual complexity of the structure, in this case, highlights the limitations introduced by common assumptions in the EF modelling of masonry structures as well. In the following, a detailed dynamic comparison with the updated FE model of the palace (\$3.2) gives further insights on the suitability of these two formulations in supporting the SHM of historical masonry structures. Since the update of both structural models targeted the same experimental modes, the comparison is extended to the entire set of the first five modes (regardless of their employment in the updating process). Moreover, to overcome some issues faced with experimental mode shapes - mainly related to spatial aliasing and due to the absence of sensors on the bell tower, $\$ 3.1-$ the two models are compared accounting for an increased number of nodes.

From a dynamic point of view, the two models show a similar ability to reproduce the ambient behaviour of the palace (see Table 3 and Table 7). Indeed, the FE model does not suffer from critical modelling simplifications (such as those related to the out-of-plane stiffness) which makes the EF updating process less straightforward. For these reasons, rather than looking at the constitutive parameters, it seems more interesting to compare directly the models' dynamic response.

For what concerns the calibration T1, the EF model does not correctly reproduce some modes of the FE model (Table 8), in particular the third mode - involving the flexural behaviour of the bell tower, Figure 3. This result is not unexpected, considering that this mode has not been included in the updating procedure. Undoubtedly, the most important difference between the two models lies in the second natural mode. In the EF model, mode 2 is mainly characterized by a local flexural behaviour of the bell tower in the $x$-direction. From a seismic point of view, this causes the activation of only a small fraction $m_{x}$ of the total dynamic mass. In the FE model, on the other hand, the mode globally involves both the main body of the palace and the bell tower, so that a significant portion of the total dynamic mass is activated.

Calibration T2 appears to overcome most of these drawbacks. First, there is a remarkably good agreement in frequencies and an acceptable correlation in mode shapes - even for modes 3 and 4 , excluded from the updating. Second, and more importantly, mode 2 configures itself 
as a global mode, with a participant mass ratio comparable with the FE one. This finding emphasizes, again, the influence of the bell tower on the global dynamic behaviour of the structure, calling for further experimental investigations specifically aimed at identifying such an interaction.

\begin{tabular}{l|ll|llll|lllll} 
& \multicolumn{2}{c|}{} & FE (2600 ton) & \multicolumn{3}{|c|}{ EF - T1 } & $(25800$ & ton $)$ & \multicolumn{4}{c}{ EF - T2 } & $(25000$ ton) \\
\hline Mode & $m_{x}$ & $m_{y}$ & $m_{x}$ & $m_{y}$ & $\Delta_{f}$ & MAC & $m_{x}$ & $m_{y}$ & $\Delta_{f}$ & MAC \\
\hline 1 & 0 & 0.80 & 0 & 0.57 & -0.011 & 0.98 & 0 & 0.58 & -0.044 & 0.98 \\
2 & 0.52 & 0 & 0.05 & 0 & 0.003 & 0.79 & 0.45 & 0 & 0.046 & 0.73 \\
3 & 0.10 & 0.01 & 0 & 0.02 & -0.105 & 0.37 & 0.01 & 0 & -0.031 & 0.65 \\
4 & 0.18 & 0 & 0 & 0 & -0.025 & 0.69 & 0.11 & 0.01 & 0.022 & 0.91 \\
5 & 0.01 & 0.02 & 0.28 & 0 & -0.082 & 0.68 & 0.08 & 0 & -0.073 & 0.74 \\
\hline
\end{tabular}

Table 8: Comparison between the updated FE model and the updated EF model T (with bell tower).

\section{CONCLUSIONS AND FUTURE PERSPECTIVES}

The paper proposes an uncommon application of the equivalent frame (EF) technique to the structural modelling of a historical masonry palace, the Consoli Palace of Gubbio, Italy. The EF modelling of such a complex and heterogeneous structure poses some challenging issues, which are discussed and specifically addressed in the work. The computational efficiency of the model allows the employment of a global search updating strategy, aimed at minimizing the differences with the experimental modal properties of the structure identified from ambient vibration tests. The results show the capability of the EF approach in describing the elastic dynamic behaviour of the structure and the need, for this structural typology, to overcome some limitations arising from simplifying modelling assumptions. The most relevant, in this case, proves to be the out-of-plane stiffness of the bearing walls, which is implicitly incorporated by the in-plane stiffness in the calibration process. The good accordance with the refined and updated Finite Element (FE) model of the structure confirms the possibility to employ the simplified EF formulation to support the model-based health monitoring of the palace. The comparison highlights, finally, the primary role played by the bell tower in determining the global dynamics of the structure. This outcome calls for further experimental investigations, which are being addressed within the Italian PRIN 2017 project DETECT-AGING through an extended dynamic, static and environmental monitoring system that has been recently deployed. The future developments will tackle the employment of both the EF and FE in the statistical simulations of post-seismic scenarios, supporting the localization and quantification of damage from vibration measurements through metamodelling. Further refinements of the EF model, aimed at the explicit description of the out-of-plane stiffness contributions of walls, are currently ongoing.

\section{ACKNOWLEDGEMENT}

This research has been supported by the Italian Ministry of Education, University and Research (MIUR) thanks to the funded project of national interest DETECT-AGING - "Degradation Effects on sTructural safEty of Cultural heriTAGe constructions through simulation and health monitorING" (Protocol No. 201747Y73L). 


\section{REFERENCES}

[1] F. Clementi, A. Formisano, G. Milani, F. Ubertini, Structural Health Monitoring of Architectural Heritage: From the past to the Future Advances. International Journal of Architectural Heritage, 15(1), 2021.

[2] P. Roca, M. Cervera, G. Gariup, L. Pelà, Structural analysis of masonry historical constructions. Classical and advanced approaches. Archives of Computational Methods in Engineering, 17(3), 299-325, 2010.

[3] A.M. D’Altri, V. Sarhosis, G. Milani, J. Rots, S. Cattari, S. Lagomarsino, E. Sacco, A. Tralli, G. Castellazzi, S. de Miranda, Modeling strategies for the computational analysis of unreinforced masonry structures: review and classification. Archives of Computational Methods in Engineering, 27, 1153-1185, 2019.

[4] S. Lagomarsino, S. Cattari, PERPETUATE guidelines for seismic performance-based assessment of cultural heritage masonry structures. Bulletin of Earthquake Engineering, 13, $13-47,2015$.

[5] E. Quagliarini, G. Maracchini, F. Clementi, Uses and limits of the Equivalent Frame Model on existing unreinforced masonry buildings for assessing their seismic risk: A review. Journal of Building Engineering, 10, 166-182, 2017.

[6] F. Ubertini, C. Gentile, A.L. Materazzi, Automated modal identification in operational conditions and its application to bridges. Engineering Structures, 46, 264-278, 2013.

[7] Simulia, Abaqus Analysis User's Manual. Volume III: Materials, Dessault Systèmes, USA, 2010.

[8] A. Kita, N. Cavalagli, F. Ubertini, Temperature effects on static and dynamic behavior of the Consoli Palace in Gubbio, Italy. Mechanical Systems and Signal Processing, 120, 180-202, 2019.

[9] N. Augenti, F. Parisi, Learning from construction failures due to the 2009 L'Aquila, Italy, earthquake. Journal of Performance of Constructed Facilities, 24(6), 536-555, 2010.

[10] S. Cattari, S. Degli Abbati, D. Ferretti, S. Lagomarsino, D. Ottonelli, A. Tralli, The seismic behaviour of ancient masonry buildings after the earthquake in Emilia (Italy) on May $20^{\text {th }}$ and 29th, 2012. Ingegneria Sismica, 29(2-3), 87-119, 2012.

[11] A. Penna, P. Morandi, M. Rota, C.F. Manzini, F. Da Porto, G. Magenes, Performance of masonry buildings during the Emilia 2012 earthquake. Bulletin of Earthquake Engineering, 12(5), 2255-2273, 2014.

[12] L. Sorrentino, S. Cattari, F. Da Porto, G. Magenes, A. Penna, Seismic behaviour of ordinary masonry buildings during the 2016 central Italy earthquakes. Bulletin of Earthquake Engineering, 17(10), 5583-5607, 2019.

[13] S. Bracchi, M. Rota, A. Penna, G. Magenes, Consideration of modelling uncertainties in the seismic assessment of masonry buildings by equivalent-frame approach. Bulletin of Earthquake Engineering, 13(11), 3423-3448, 2015.

[14] D. Ottonelli, C. Marano, C. Manzini, B. Calderoni, S. Cattari, A comparative study on a complex URM building. Part I: sensitivity of the seismic response to different modelling options in the equivalent frame models. Bulletin of Earthquake Engineering, 2021. Submitted to the S.I.: URM nonlinear modelling - Benchmark project. 
[15] S. Lagomarsino, A. Penna, A. Galasco, S. Cattari, TREMURI program: an equivalent frame model for the nonlinear seismic analysis of masonry buildings. Engineering structures, 56, 1787-1799, 2013.

[16] N. Augenti, Seismic behaviour of irregular masonry walls. Proceedings of the 1st European Conference on Earthquake Engineering and Seismology (ECEES), Geneva, Switzerland, 3-8 September, 2006.

[17] M. Dolce, Schematizzazione e modellazione degli edifici in muratura soggetti ad azioni sismiche. Industria delle costruzioni, 25(242), 44-57, 1991 (in Italian).

[18] F.L. Moon, T. Yi, R.T. Leon, L.F. Kahn, Recommendations for seismic evaluation and retrofit of low-rise URM structures. Journal of Structural Engineering, 132(5), 663-672, 2006.

[19] S. Cattari, D. Camilletti, A.M. D'Altri, S. Lagomarsino, On the use of continuum Finite Element and Equivalent Frame models for the seismic assessment of masonry walls, Journal of Building Engineering, 2021. https://doi.org/10.1016/j.jobe.2021.102519

[20] F. Parisi, N. Augenti, Seismic capacity of irregular unreinforced masonry walls with openings, Earthquake Engineering and Structural Dynamics, 42(1), 101-121, 2013.

[21] M. Berti, L. Salvatori, M. Orlando, P. Spinelli, Unreinforced masonry walls with irregular opening layouts: reliability of equivalent-frame modelling for seismic vulnerability assessment. Bulletin of Earthquake Engineering, 15(3), 1213-1239, 2017.

[22] R. Siano, V. Sepe, G. Camata, E. Spacone, P. Roca, L. Pelà, Analysis of the performance in the linear field of equivalent-frame models for regular and irregular masonry walls. Engineering Structures, 145, 190-210, 2017.

[23] B. Calderoni, E.A. Cordasco, C. Musella, A. Sandoli, La modellazione delle pareti murarie in relazione alle irregolarità geometriche: problemi aperti. Proceedings of XVII ANIDIS Conference, Pistoia, Italy, 17-21 September, 2017. (in Italian)

[24] R. Siano, P. Roca, G. Camata, L. Pelà, V. Sepe, E. Spacone, M. Petracca, Numerical investigation of non-linear equivalent-frame models for regular masonry walls. Engineering Structures, 173, 512-529, 2018.

[25] D. Camilletti, S. Cattari, S. Lagomarsino, In plane seismic response of irregular URM walls through Equivalent Frame and Finite Element models, Proceedings of $16^{\text {th }}$ European Conference on Earthquake Engineering (ECEE), Thessaloniki, Greece, 18-21 June, 2018.

[26] D. Camilletti, Equivalent Frame modelling of URM buildings: numerical validation and rules. PhD thesis, University of Genoa, Genoa, Italy, 2019.

[27] S. Cattari, G. Magenes, Benchmarking the software packages to model and assess the seismic response of unreinforced masonry existing buildings through nonlinear static analyses. Bulletin of Earthquake Engineering, 2021. https://doi.org/10.1007/s10518-02101078-0

[28] MIT 2019, Ministry of Infrastructures and Transportation, Circ. C.S.LL.PP. No. 7 of $21 / 1 / 2019$. Istruzioni per l'applicazione dell'aggiornamento delle norme tecniche per le costruzioni di cui al Decreto Ministeriale 17 Gennaio 2018. G.U.S.O. No. 35 of 11/2/2019. (in Italian) 
[29] S. Cattari, S. Resemini, S. Lagomarsino, Modelling of vaults as equivalent diaphragms in 3D seismic analysis of masonry buildings. In Structural analysis of historic construction: preserving safety and significance, Two Volume Set, 537-544, 2008. CRC press.

[30] D. Sivori, M. Lepidi, S. Cattari, Structural identification of the dynamic behavior of floor diaphragms in existing buildings. Smart Structures and Systems, 27(2), 173, 2021.

[31] D. Sivori, M. Lepidi, S. Cattari, Ambient vibration tools to validate the rigid diaphragm assumption in the seismic assessment of buildings. Earthquake Engineering and Structural Dynamics, 49(2), 194-211, 2020.

[32] B. Jaishi, W.X. Ren, Structural finite element model updating using ambient vibration test results. Journal of structural engineering, 131(4), 617-628, 2005.

[33] R.J. Allemang, The modal assurance criterion-twenty years of use and abuse. Sound and vibration, 37(8), 14-23, 2003.

[34] S. Cattari, S. Degli Abbati, S. Alfano, A. Brunelli, F. Lorenzoni, F. Da Porto, Dynamic calibration and seismic validation of numerical models of URM buildings through permanent monitoring data. Earthquake Engineering and Structural Dynamics, 2021. Accepted for publication. 